\title{
Photosensitive post tuning of chalcogenide photonic crystal waveguides
}

\author{
Michael W. Lee, Christian Grillet, Cameron L.C. Smith, David J. Moss*, \\ Benjamin J. Eggleton
}

Centre for Ultrahigh-bandwidth Devices for Optical Systems (CUDOS)

School of Physics, University of Sydney, Sydney, NSW, 2006, Australia grillet@physics.usyd.edu.au

* Now with Institut national de la recherche scientifique (INRS)

Énergie, Matériaux et Télécommunications, Université du Québec

1650 boulevard Lionel-Boulet, Varennes, Québec, Canada, J3X-1S2

Darren Freeman, Barry Luther-Davies, Steve Madden, Andrei Rode

Centre for Ultrahigh bandwidth Devices for Optical Systems, Laser Physics Centre, Australian National University,

Canberra, Australia

Yinlan Ruan

Center of Expertise in Photonics, School of Chemistry and Physics, University of Adelaide, Adelaide, Australia

\section{Yong-hee Lee}

Nanolaser Laboratory, Department of Physics, Korea Advanced Institute of Science and Technology, Daejeon 305 701, Korea

\begin{abstract}
We present experimental results on post-tuning the dispersion of a two-dimensional photonic crystal waveguide made from $\mathrm{Ge}_{33} \mathrm{As}_{12} \mathrm{Se}_{55}$ chalcogenide glass by exploiting the material photosensitivity to nearbandgap light. The change in the refractive index and volume of the material in response to exposure to $633 \mathrm{~nm}$ light resulted in a shift of more than $5 \mathrm{~nm}$ in the resonant coupling wavelength between a tapered optical fiber and the modes of a W1 waveguide. This represents a first proof of principle demonstration of the photosensitive post-tuning of a planar photonic crystal device.
\end{abstract}

(C)2007 Optical Society of America

OCIS code: (230.3120) Optical devices; Integrated optics devices, (230.7370) Optical devices; waveguides, (060.1810) Fiber optics and optical communications; Couplers, switches, and multiplexers, (160.5320) Materials; Photorefractive materials

\section{References and links}

1. J-M. Lourtioz, H. Benisty, V. Berger, J-M. Gerard, D. Maystre, and A. Tchelnokov, Photonic Crystals: Towards Nanoscale Photonic Devices (Springer, 2005).

2. H. M. H. Chong, and R. Delarue, "Tuning of photonic crystal waveguide microcavity by thermooptic effect," IEEE Phot. Tech. Lett. 16, 1528-1530 (2004).

3. R. Ferrini, J. Martz, L. Zuppiroli, B. Wild, V. Zabelin, L. A. Dunbar, R. Houdré, M. Mulot, and S. Anand, "Planar photonic crystals infiltrated with liquid crystals: optical characterization of molecule orientation," Opt. Lett. 31, 1238-1240 (2006).

4. D. Erickson, T. Rockwood, T. Emery, A. Sherer, and D. Psaltis, "Nanofluidic tuning of photonic crystal circuits," Opt. Lett. 31, 59-61 (2006).

5. D. Dalacu, S. Frédérick, P. J. Poole, G. C. Aers, and R. L. Williams, "Postfabrication fine-tuning of photonic crystal microcavities in InAs/InP quantum dot membranes," Appl. Phys. Lett. 87, 151107 (2005).

6. W. Park, J-B. Lee, "Mechanically tunable photonic crystal structure," Appl. Phys. Lett. 85, 4845-4847 (2004).

7. I. Marki, M. Salt, and H. P. Herzig, "Tuning the resonance of a photonic crystal microcavity with an AFM probe,” Opt. Express 14, 2969-2978 (2006). 
8. J. M. Gerard and B. Gayral, "Toward high-efficiency quantum-dot single photon sources," in Quantum Dots, Nanoparticles, and Nanoclusters, D. L. Huffaker and P. Bhattacharya, eds, Proc. SPIE 5361, 88-95 (2004).

9. D. Englund, D. Fattal, E. Waks, G. Solomon, B. Zhang, T. Nakaoka, Y. Arakawa, Y. Yamamoto, and J. Vuckovic, "Controlling the spontaneous emission rate of single quantum dots in a two-dimensional photonic crystal,” Phys. Rev. Lett. 95, 013904 (2005).

10. A. Zakery, S. R. Elliot, "Optical properties and applications of chalcogenide glasses: a review," J. NonCryst. Solids 330, 1-12 (2003).

11. C. Grillet, C. Smith, D. Freeman, S. Madden, B. Luther-Davies, E. Magi, D. Moss, and B. Eggleton, "Efficient coupling to chalcogenide glass photonic crystal waveguides via silica optical fiber nanowires," Opt. Express 14, 1070-1078 (2006).

12. S. Ramachandran, and S. Bishop, "Photoinduced integrated-optics in rapid thermally annealed chalcogenide glasses,” IEEE J. Sel. Top. Quantum Electron. 11, 260-270 (2005).

13. R. Decorby, N. Ponnampalam, M. Pai, H. Nguyen, P. Dwivedi, T. Clement, C. Haugen, J. McMullin, and S. Kasap, “High index contrast waveguides in Chalcogenide glass and polymer," IEEE J. Sel. Top. Quantum Electron. 11, 539-546 (2005).

14. V. Lyubin, M. Klebanov, A. Feigel, and B. Sfez, "Films of chalcogenide glassy semiconductors: New phenomena and new applications," Thin Solid Films 459, 183-186 (2004).

15. M. Shokooh-Saremi, V. Taeed, I. Littler, D. Moss, B. Eggleton, Y. Ruan, and B. Luther-Davies, "Ultrastrong, well-apodised Bragg gratings in Chalcogenide rib waveguides," Electron. Lett. 41, 13-14 (2005)

16. T. Sudoh, Y. Nakano, and K. Tada, "Wavelength trimming technology for multiple-wavelength distributed feedback laser array by photo-induced refractive index change," Electron. Lett. 33, 216-217 (1997).

17. S. Song, S. Howard, Z. Liu, A. Dirisu, C. Gmachl, and C. Arnold, "Mode tuning of quantum cascade lasers through optical processing of Chalcogenide glass claddings," Appl. Phys. Lett. 89, 041115 (2006).

18. A. Zakery, Y. Ruan, A. V. Rode, M. Samoc, and B. Luther-Davies, "Low-loss waveguides in ultrafast laser-deposited As_2S_3 chalcogenide films ," J. Opt. Soc. Am. B 20, 1844-1852 (2003).

19. T. T. Nang, M. Okuda and T. Matsushita, "Photo-induced absorption change in some Se-based glass alloy systems,” Phys. Rev. B 19, 947-955 (1979).

20. K. Tanaka, "Reversible photostructural change: Mechanisms, properties and applications," J. Non-Cryst. Solids 35-36, 1023-1034 (1980)

21. S. R. Elliott, "A unified model for reversible photostructural effects in chalcogenide glasses," J. Non-Cryst. Solids 81, 71-98 (1986)

22. Y. Ruan, M. Kim, Y. Lee, B. Luther-Davies, and A. Rode, "Fabrication of high-Q chalcogenide photonic crystal resonators by e-beam lithography," submitted to Applied Physics Letters.

23. D. Freeman, S. Madden, and B. Luther-Davies,"Fabrication of planar photonic crystals in a chalcogenide glass using a focused ion beam,” Opt. Express 13, 3079-3086 (2005).

24. C. Grillet, D. Freeman, B. Luther-Davies, S. Madden, R. McPhedran, D. J. Moss, M. J. Steel, and B. J. Eggleton, "Characterization and modeling of Fano resonances in chalcogenide photonic crystal membranes," Opt. Express 14, 369-376 (2006).

25. Y. K. Lizé, E. C. Mägi, V. G. Ta'eed, J. A. Bolger, P. Steinvurzel, and B. J. Eggleton, "Microstructured optical fiber photonic wires with subwavelength core diameter," Opt. Express 12, 3209-3217 (2004).

26. S. Madden, "Investigation into the photosensitivity of AMTIR-1 films," Laser Physics Centre, Australian National University (personal communication, 2006).

27. A. Zakery and M. Hatami, "Nonlinear optical properties of pulsed-laser-deposited GeAsSe films and simulation of a nonlinear directional coupler switch," J. Opt. Soc. Am. B 22, 591-597 (2005)

28. T. Igo, Y. Noguchi and H. Nagai, "Photoexpansion and 'thermal contraction' of amorphous chalcogenide glasses,” Appl. Phys. Lett. 25, 193-194 (1974)

29. B. Song, S. Noda, T. Asano, and Y. Akahane, "Ultra-high-Q photonic double heterostructure nanocavity," Nature Mater. 4, 207-210 (2005).

30. S. Tomljenovic-Hanic, M. Steel, C. de Sterke, and D. Moss, "High-Q cavities in photosensitive photonic crystals," Opt. Lett. Accepted in press.

\section{Introduction}

Planar Photonic Crystals (PhC), which consist of a thin high index dielectric slab patterned with a 2D periodic array of air holes, are now recognized as a promising platform for achieving light control in a planar integrated circuit. Their ability to confine light at the wavelength scale has led to the demonstration of unprecedented compact photonic devices for integrated optical circuits [1]. However, in order to achieve desirable functionalities careful engineering and high accuracy fabrication is required. Although advances both in numerical 
designs and nanofabrication techniques have enabled the development of more intricate photonic crystal systems, the required level of fabrication accuracy remains a limiting factor.

Post trimming of the properties of individual components is thus highly attractive, not only to relax the fabrication tolerances but also to allow individual components to be optimized for different environments or applications. Tuning of photonic crystal properties has previously been achieved through thermo-optic approaches [2], liquid crystal infusion [3], integration of nanofluidic circuitry [4], post-process chemical treatments [5], mechanical deformation [6] or mechanical perturbation of the electro-magnetic environment with an Atomic Force Microscopy (AFM) tip [7]. Although these approaches can provide enough tuning to modify the optical properties of very compact devices, they generally lack the highly desirable ability to induce changes locally. In particular, single Quantum Dot (QD) devices based on cavity QED require fine tuning of the photonic crystal cavity resonance in relation to the emission wavelength of the QD [8,9].

In this paper we demonstrate a novel post-process tuning technique which utilizes the photosensitivity of chalcogenide glass [10] to modify the optical properties of a planar photonic crystal device. A W1 PhC waveguide was exposed to $633 \mathrm{~nm}$ light at an intensity of $1.3 \mathrm{~W} / \mathrm{cm}^{2}$. The resulting change in the dispersion of the modes of the waveguide was detected in situ using an evanescent probing technique [11], and a shift of $5 \mathrm{~nm}$ in the resonant coupling wavelength was observed. We show that the photoinduced change in the photonic crystal grows as a saturating exponential with increasing exposure.

Previously, the chalcogenide photosensitivity has been successfully utilized for the creation of directly written waveguides [12, 13, 14], strong Bragg gratings [15] and for posttuning of optical components such as distributed feedback lasers [16] and quantum cascade lasers [17]. The photosensitivity of the chalcogenides is known to arise from structural rearrangements induced by the absorption of light at frequencies near the absorption bandedge of the material [18]. These structural rearrangements lead to changes in the properties of the glass such as the refractive index and density. Some of the various photoinduced phenomena are reviewed in [10]. The magnitude and sign of these photoinduced changes can be highly dependant on the chemical composition of the glass [19] and on the processing history of the sample [20]. Furthermore, these changes may be either reversible or irreversible, with irreversible changes resulting from "photo-annealing" of thin films deposited in a non-equilibrium state [21], and reversible changes resulting from structural rearrangement into a quasi-stable state upon exposure [20,21].

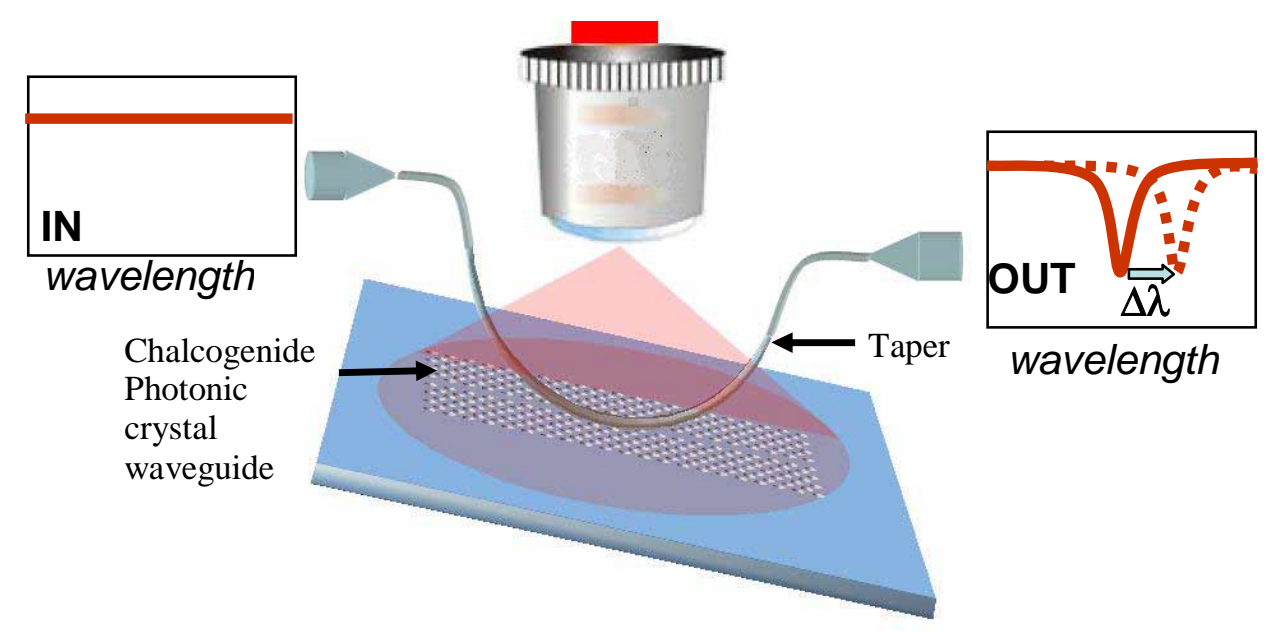

Fig. 1. A schematic diagram showing the principle of the photosensitive post tuning of a chalcogenide photonic crystal waveguide. 


\section{Principle}

Figure 1 shows the principle of the photosensitive post-tuning experiment. The resonant coupling wavelength from the fiber taper to the modes of the chalcogenide glass $\mathrm{PhC}$ waveguide was monitored by measuring the transmission spectrum through the taper with an Optical Spectrum Analyzer (OSA). The dips in the transmission spectrum are associated with coupling to the modes of the $\mathrm{PhC}$ waveguide. The photoinduced change in the $\mathrm{PhC}$ was observed by monitoring the shift in wavelength of these dips during the exposure of the $\mathrm{PhC}$ sample to $633 \mathrm{~nm}$ light.

\section{Photonic crystal waveguide design and device fabrication}

Figure 2 shows an optical microscope image and a Scanning Electron Microscope (SEM) image of the photonic crystal structure used in this experiment. The structure under test consists of a $\sim 70 \mu \mathrm{m}$ "W1" waveguide, i.e. a missing row of holes along the $\Gamma-\mathrm{K}$ direction of a triangular lattice of air holes in a chalcogenide membrane.

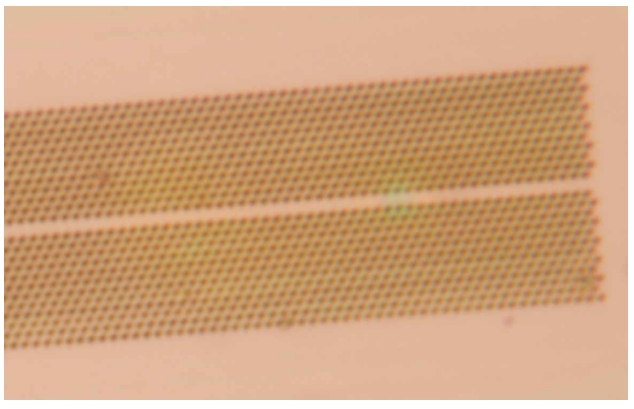

(a)

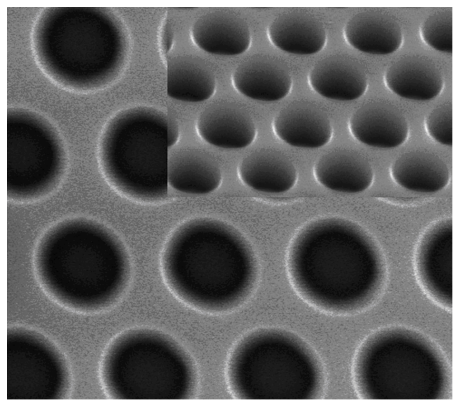

(b)

Fig. 2. (a) Optical microscope image of the $\mathrm{W} 1 \mathrm{PhC}$ waveguide used in this experiment. (b) Scanning electron microscope image. Hole pitch is $550 \mathrm{~nm}$.

Details of the fabrication process are presented elsewhere [22] and are summarized briefly here. First, a 300nm thick film of $\mathrm{Ge}_{33} \mathrm{As}_{12} \mathrm{Se}_{55}$ (AMTIR-1) chalcogenide glass [23, 24] was deposited by Ultrafast Pulsed Laser Deposition (UPLD) onto an oxidised silicon wafer. The sample was then coated with a thin layer of polymer photoresist (PMMA). The PhC structure was written into the photoresist by electron beam lithography and transferred into the chalcogenide layer using Chemically Assisted Ion Beam Etching (CAIBE). Finally, a free standing suspended $\mathrm{PhC}$ structure was formed by wet etching the oxidised silicon layer beneath the chalcogenide film. The resulting $300 \mathrm{~nm}$ thick suspended membrane had a nominal refractive index of 2.7 and hence corresponded to a thickness of $\lambda / 2 \mathrm{n}$ close to $\lambda=1.55$ $\mu \mathrm{m}$, ensuring that only the fundamental mode is vertically confined in this structure. A lattice parameter of $550 \mathrm{~nm}$ and hole radius of $165 \mathrm{~nm}$ were chosen in order to open up a photonic band gap for the TE-like modes (E field mainly lying in the plane of the slab) near 1550nm. The chalcogenide $\mathrm{PhC}$ membrane was kept in its as deposited state (not thermally annealed) and was protected from ambient light prior to the experiment.

\section{Post-trimming photosensitive experiment}

A schematic diagram of the "post-trimming" experiment is shown in Fig. 3. It consists of; (i) the evanescent coupling setup which was used to probe the modes of the $\mathrm{PhC}$ waveguide, allowing the photoinduced change to be monitored and; (ii) the "writing" system, which consisted of a $633 \mathrm{~nm}$ linearly polarized $0.8 \mathrm{~mW} \mathrm{CW}$ Helium Neon laser source, a lens and a beamsplitter. 


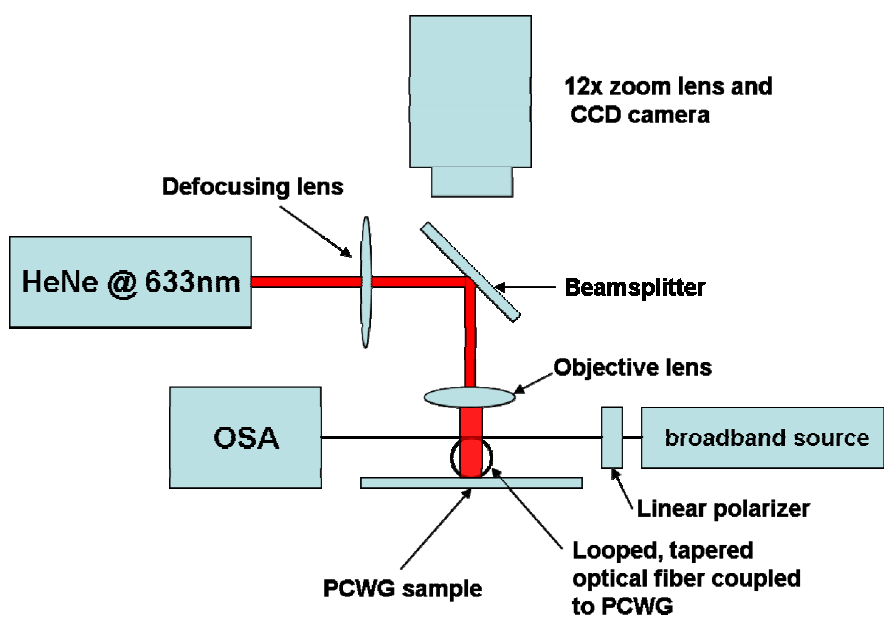

Fig. 3. A schematic diagram of the experimental setup for both coupling into the Photonic Crystal Waveguide (PCWG) using evanescent coupling and photosensitive post-processing the $\mathrm{PhC}$ sample.

It consists of; (i) the evanescent coupling setup which was used to probe the modes of the $\mathrm{PhC}$ waveguide, allowing the photoinduced change to be monitored and; (ii) the "writing" system, which consisted of a $633 \mathrm{~nm}$ linearly polarized $0.8 \mathrm{~mW} \mathrm{CW}$ Helium Neon laser source, a lens and a beam splitter.

The evanescent coupling system is described in detail in [11]. Evanescent coupling is a phase-matched technique where, in this case, the evanescent field of a tapered optical fiber is used to couple light into the PhC waveguide brought into close proximity with the tapered fiber (Fig. 4. a and b). The condition for coupling to occur is that the taper mode and $\mathrm{PhC}$ waveguide mode must possess some spatial overlap, as well as sharing the same momentum. This occurs where the taper dispersion curve intersects the dispersion curve of the waveguide mode. Figure $4 \mathrm{~b}$ shows the calculated band structure of a W1 waveguide with the same parameters as the sample used in this work. Overlaid on this is the dispersion curve corresponding to the mode of a $1.3 \mu \mathrm{m}$ diameter fiber taper. The band structures were calculated with the RSoft bandSOLVE program which uses the method of plane wave expansion to calculate the bands.

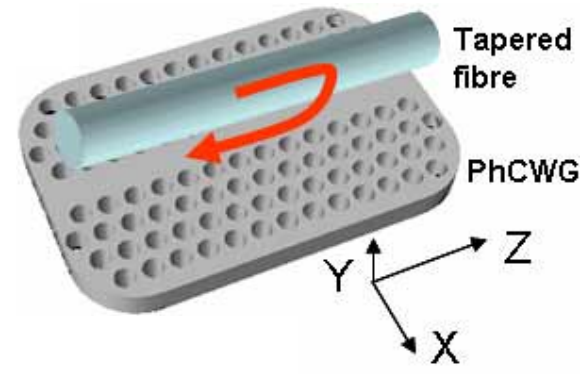

(a)

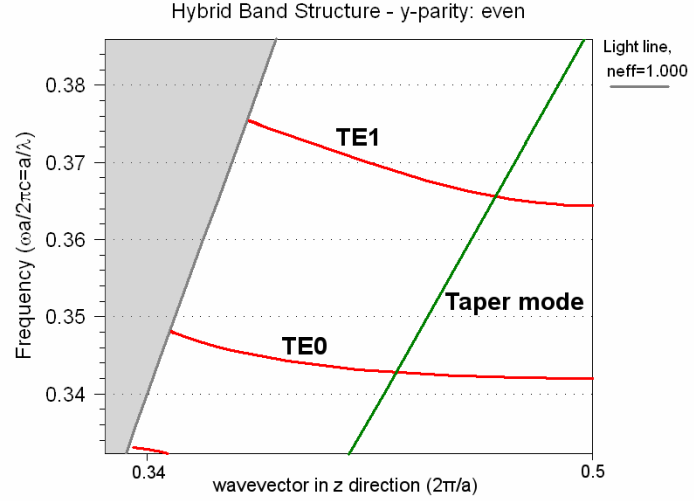

(b)

Fig. 4. a) Schematic showing the coupling from a tapered fibre to $\mathrm{PhC}$ waveguide, b) Band structure of the W1 waveguide used in this work (red) overlaid with the dispersion curve of the tapered fiber in green.

$\# 77808$ - \$15.00 USD

(C) 2007 OSA
Received 5 December 2006; revised 22 January 2007; accepted 26 January 2007 5 February 2007 / Vol. 15, No. 3 / OPTICS EXPRESS 1281 
The tapered fibers were fabricated using a procedure reported previously [25], and for this work the fiber taper was made from standard single mode fiber. After tapering, the fibre was formed into a loop in the waist region in order to localize the interaction region between the taper and the photonic crystal waveguide. The taper used in this work was estimated to have a waist diameter of $\sim 1.3 \mu \mathrm{m}$ and a loop diameter of $\sim 200 \mu \mathrm{m}$. The loss through the taper was $\sim 1 \mathrm{~dB}$ in the wavelength range of $1550 \mathrm{~nm}-1620 \mathrm{~nm}$. To achieve evanescent coupling, the taper was aligned above and parallel to the photonic crystal and then moved into direct contact so that the relative alignment of the taper with the waveguide, and hence the coupling, remained fixed throughout the experiment. A broadband light source (Hewlet Packard 83437A, a four ELED light source) was launched into the taper through a polarizer to select TE polarization and the output end of the fiber was connected to an OSA (Agilent 86140B) where the transmission spectrum through the taper was measured. The polarization was adjusted by placing the taper onto the bulk $\mathrm{PhC}$ region and then adjusting the polarizer to minimize the loss in the transmission of the taper. Since the $\mathrm{PhC}$ has a bandgap in the TE modes at the frequencies of interest, loss is associated with coupling to TM modes of the bulk $\mathrm{PhC}$ and hence minimizing the loss results in the TE polarization at the taper waist.

Figure 5 shows a typical transmission spectrum through the fiber taper when aligned and in contact with the PhC waveguide. Resonances corresponding to coupling to both the fundamental $\mathrm{W} 1$ guided mode $\left(\mathrm{TE}_{0}\right)$ and the first higher order mode $\left(\mathrm{TE}_{1}\right)$ can be identified. The coupling wavelengths are in good agreement with the predicted values based on band structure calculations. The small difference between the predicted coupling wavelengths, shown by the dashed line, and the experimentally measured values is due to the uncertainty in the $\mathrm{PhC}$ air hole spacing and radius, slab thickness and refractive index as well as the dispersion of the chalcogenide which is not taken into account in the modeling. The transmission dip associated with coupling to the fundamental $\mathrm{TE}_{0}$ mode was $\sim 6 \mathrm{~dB}$, which equates to $\sim 75 \%$ of the light being coupled from the taper into the waveguide. This lower coupling efficiency compared to [11] is expected because of the looped taper shape used in this experiment which results in a substantially smaller coupling length.

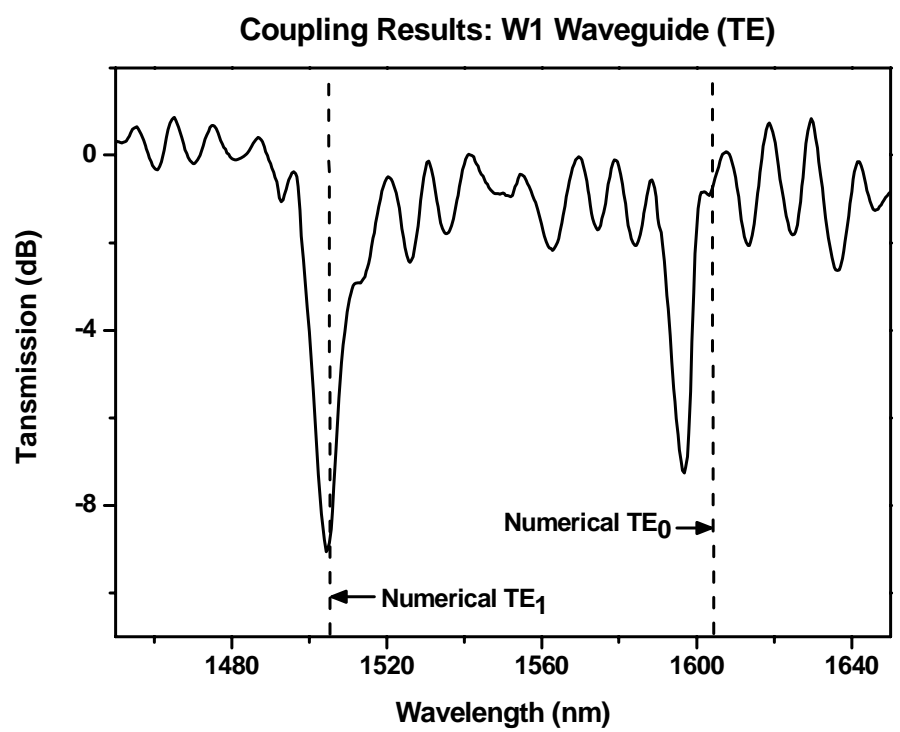

Fig.5. Transmission spectrum through the tapered fiber for coupling to the W1 waveguide, with the fiber in direct contact with the waveguide. Dashed lines represent numerically predicted values for the $\mathrm{TE}_{0}$ and $\mathrm{TE}_{1}$ coupling resonances.

In order to expose the sample to actinic light, the laser source was focused on the sample via a lens and beam splitter (see Fig. 3). A wavelength of $633 \mathrm{~nm}$ was chosen because the photon energy is close to but slightly below the electronic band edge for AMTIR-1, where 
these glasses are generally photosensitive [14]. The beamsplitter was used to direct the beam towards the sample at normal incidence, while allowing in-situ imaging of the sample. A defocusing lens was introduced in order to control the laser spot size at the sample. The defocusing lens was positioned to give a spot diameter of $\sim 280 \mu \mathrm{m}\left(1 / \mathrm{e}^{2}\right.$ Gaussian spot). Given that the photonic crystal waveguide is $\sim 70 \mu \mathrm{m}$ long, this means that the incident intensity varied by less than $10 \%$ across the sample. The power measured at the sample was $400 \mu \mathrm{W}$ corresponding to a peak intensity of $1.3 \mathrm{~W} / \mathrm{cm}^{2}$ for the Gaussian laser spot. After obtaining an optimal coupling into the $\mathrm{PhC}$ waveguide, the $\mathrm{HeNe}$ laser was turned on and the position of the laser spot adjusted so that it was centered on the $\mathrm{PhC}$ structure. During alignment the laser was strongly attenuated $(40 \mathrm{~dB})$. The sample was exposed for a period of $\sim 5$ hours and the transmission spectrum through the taper was recorded at one minute intervals during this time. Further, the sample was monitored for a period of $\sim 5$ days following the exposure to determine whether the observed change was stable. The experiment was conducted at room temperature with the ambient room light switched off.

\section{Results and discussion}

Figure 6 shows the transmission spectrum of the fiber-taper due to coupling to the fundamental $\mathrm{PhC}$ waveguide mode for different exposure times. The resonance associated with coupling to the $\mathrm{TE}_{0}$ mode was observed to shift to longer wavelengths with increasing exposure. The same trend was observed for the higher order $\mathrm{TE}_{1} \mathrm{PhC}$ waveguide mode. In addition, we observed an increase in the coupling strength with exposure.

$\mathrm{TE}_{0}$ Coupling Spectra During Exposure

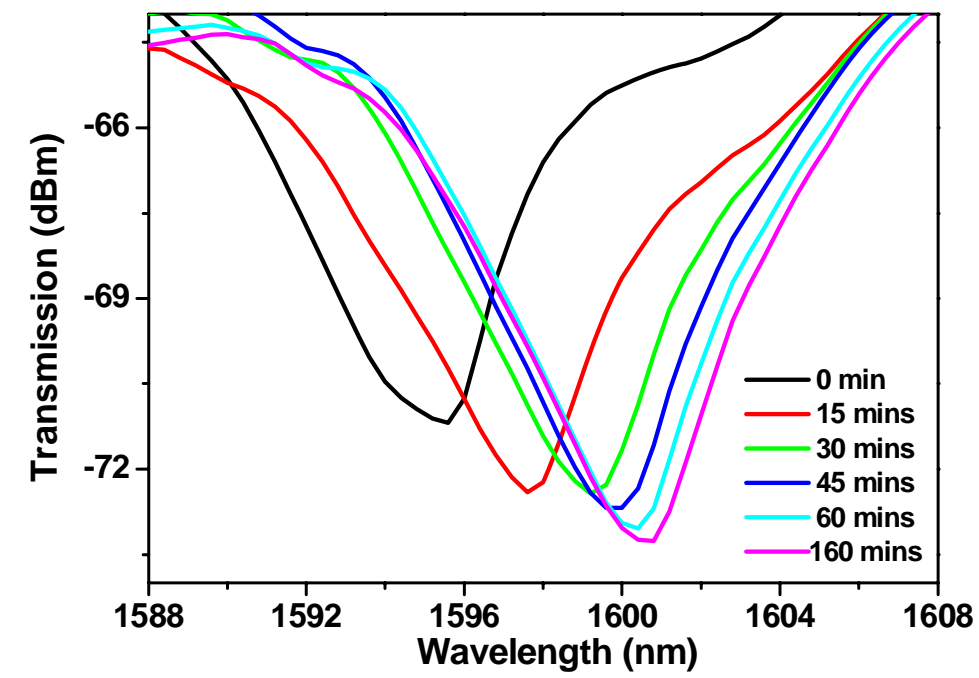

Fig. 6. Photosensitive tuning of the $\mathrm{TE}_{0}$ mode during the exposure. The resonance dip is plotted for a range of times during the exposure.

Figure 7 shows a plot of the resonant wavelength versus exposure fluence, which clearly displays saturation behaviour at higher fluences. The circles in the graph are experimental data points whilst the curve is an empirical fit to an exponential curve. The maximum wavelength shift was $5.2 \mathrm{~nm} \pm 0.4 \mathrm{~nm}$ whilst the maximum increase in the resonance depth was $\sim 2 \mathrm{~dB}$. 
Shift of $\mathrm{TE}_{0}$ Coupling Wavelength During Exposure

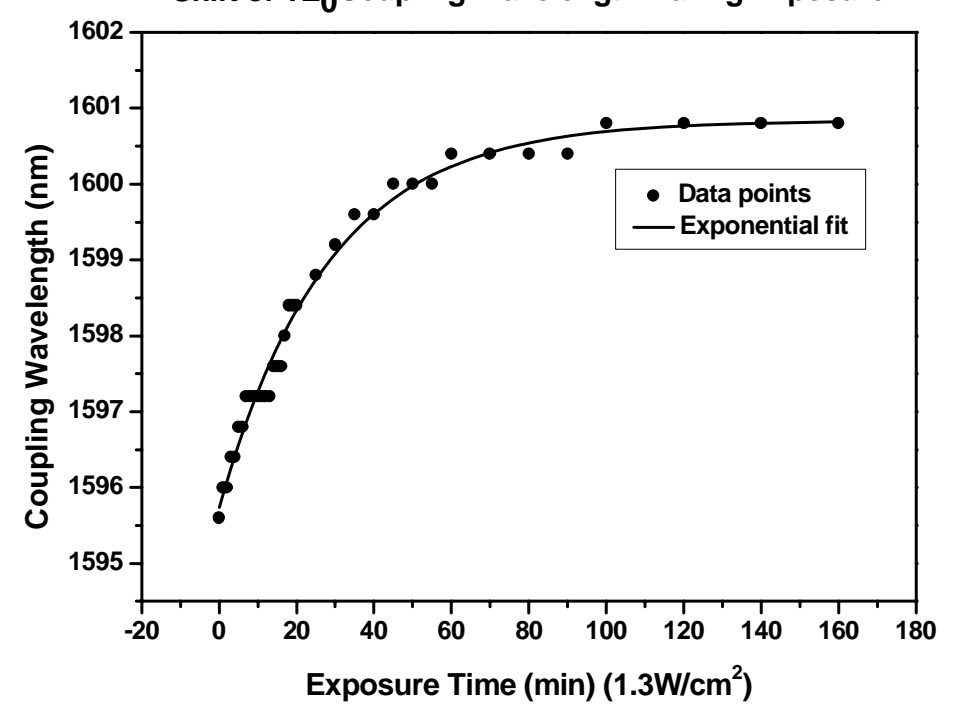

Fig. 7. Shift in coupling wavelength versus exposure fluence at $633 \mathrm{~nm}$ of the $\mathrm{PhC} \mathrm{TE}_{0}$ waveguide mode.

The exact cause of the observed increase in coupling strength is still under investigation but it is likely to be related to a modification of the waveguide mode field profile resulting from changes in the waveguide dispersion curve. In principle, one would expect that in modifying the group index of the $\mathrm{PhC}$ waveguide mode, the overlap with the taper mode, and hence the coupling strength, could change. The shift in the coupling wavelength of the higher order waveguide mode follows the same trend as for the fundamental mode and during the exposure there was no relative shift between the two modes, i.e. the wavelength difference remained constant to within the resolution of the OSA.

Preliminary investigations into the photosensitivity of unpatterened AMTIR-1 films [26] at $633 \mathrm{~nm}$ have shown a decrease in the material refractive index and a volume expansion. These results are consistent with previous work by Zakery et al. [27], who reported a refractive index decrease in AMTIR-1 due to exposure, and Igo et al. [28], who reported photoexpansion in a range of As-Se-Ge glasses. For the $\mathrm{PhC}$ waveguide, a refractive index decrease results in a shift of the waveguide modes to shorter wavelengths. Our calculations indicate that a wavelength shift of $\sim 5 \mathrm{~nm}$ is obtained with an index change of 0.01 , and that this change occurs linearly in the region of interest. Conversely, expansion of the $\mathrm{PhC}$ causes a shift towards longer wavelengths, a $\sim 5 \mathrm{~nm}$ wavelength shift of the $\mathrm{TE}_{0}$ mode is obtained for $0.31 \%$ material expansion. Thus we attribute the observed wavelength shift to a combination of these two competing effects. However, the material expansion has the bigger effect in this case leading to the observed resonance shift to longer wavelengths. Work is in progress to resolve the magnitude of each contribution to the resonance shift.

For many potential applications, the ability to tune the resonance towards longer wavelengths in a controlled manner is particularly relevant. For instance, the double heterostructure cavity suggested by Noda et al. [29] relies on precise engineering of the lattice constants along a line defect. Light is confined to the central region (larger lattice constant) due to the differences between the mode-gap frequencies along this line defect. In the same way, it should be possible to create a double heterostructure cavity by exposing a small region of a W1 in chalcogenide photonic crystal. Based on band structure calculations, a 5nm shift is equivalent to the band shift that would be induced by an increase in the refractive index of 0.01 . Results reported in [30] show that this should lead to a cavity Q $\sim 30000$. In order to reach the maximum $\mathrm{Q}$ predicted theoretically, a $\sim 20 \mathrm{~nm}$ shift would be required. Future 
studies into different techniques or different chalcogenide glass compositions may increase the tuning range.

Another area where the photosensitivity of chalcogenide glass could prove to be highly practical is single quantum dot devices based on cavity QED, such as a single photon source. This technique offers a way to align the cavity resonant wavelength with the QD emission wavelength - an important challenge since enhancing the QD SE rate via the Purcell factor of the cavity mode requires critical spectral matching between the single QD and the cavity mode. The ability to tune the cavity resonant wavelength, via a photosensitive chalcogenide cladding applied on top of the PhC device for instance, in order to match it to the QD emission appears highly promising.

\section{Conclusions}

We present the first experimental demonstration of photosensitive post-tuning of a planar photonic crystal device. We use the material photosensitivity of $\mathrm{Ge}_{33} \mathrm{As}_{12} \mathrm{Se}_{55}$ (AMTIR-1) chalcogenide glass to $633 \mathrm{~nm}$ light in order to modify the resonant coupling wavelength of a $\mathrm{W} 1$ photonic crystal waveguide by up to $\sim 5 \mathrm{~nm}$ towards longer wavelengths. The wavelength shift showed a saturating exponential growth trend with increasing exposure, and this suggests that a high degree of control over the photosensitive tuning process should be possible. This work demonstrates that this post-processing technique is highly promising for applications where fine post-tuning of existing structures is required, such as for single photon source applications or for directly photo-writing resonant cavity structures.

\section{Acknowledgments}

This work was produced with the assistance of the Australian Research Council under the ARC Centres of Excellence program. CUDOS (the Centre for Ultrahigh bandwidth Devices for Optical Systems) is an ARC Centre of Excellence.

\#77808 - \$15.00 USD

(C) 2007 OSA
Received 5 December 2006; revised 22 January 2007; accepted 26 January 2007 5 February 2007 / Vol. 15, No. 3 / OPTICS EXPRESS 1285 